Check for updates

Cite this: Chem. Commun., 2019, 55,7667

Received 16th May 2019, Accepted 7th June 2019

DOI: $10.1039 / c 9 c c 03791 h$

rsc.li/chemcomm

\title{
Covalently bound monolayer patterns obtained by plasma etching on glass surfaces $\dagger$
}

\author{
Stan B. J. Willems, (D) abc Laura M. I. Schijven, (D) anton Bunschoten, (D) ac \\ Fijs W. B. van Leeuwen, ${ }^{\text {ac }}$ Aldrik H. Velders (D) ac and Vittorio Saggiomo (D) *a
}

\begin{abstract}
Micropatterns of $\beta$-cyclodextrin ( $\beta-C D)$ monolayers on glass are obtained by using a plasma etching approach with polydimethylsiloxane (PDMS) stamps. This simple and versatile approach provides a promising alternative to current techniques for creating patterns of covalently bound molecules. It is also possible to fabricate sub-10 $\mu \mathrm{m}$ sized features.
\end{abstract}

For decades, molecular monolayers on surfaces have been used for the fabrication of functional materials. Surfaces have been modified to tune both physical properties, e.g. wettability, and chemical properties, e.g. supramolecular interactions. ${ }^{1}$ The resulting monolayer assemblies on glass have been extensively studied with a plethora of molecular sensors. One way to study monolayer assembly is through using bright field or fluorescence microscopy. ${ }^{2-4}$ Within microscopy, spatial control of surface modification became important to allow validation within a single field of view. Therefore patterning of molecules on surfaces was introduced by Whitesides et al. in the form of microcontact printing $(\mu \mathrm{CP}){ }^{5} \mu \mathrm{CP}$ is a soft lithographic technique wherein polydimethylsiloxane (PDMS) stamps are used for patterning surfaces, similar to Gutenberg's printing press. The patterned PDMS stamps are generally made from a silicon master wafer which contains features fabricated through UV-lithography. Importantly, this form of patterning allows positive and negative experimental controls to be placed in one field of view, which is a feature that promotes qualification of analytes. However, a major disadvantage within $\mu \mathrm{CP}$ is that the inking and patterning step requires a lengthy process of trial and error, due to issues such as solvent compatibility of

\footnotetext{
${ }^{a}$ Laboratory of BioNanoTechnology, Wageningen University and Research, Bornse Weilanden 9, 6708 WG Wageningen, The Netherlands.

E-mail: vittorio.saggiomo@wur.nl

${ }^{b}$ Wetsus, European Centre of Excellence for Sustainable Water Technology, Oostergoweg 9, 8911 MA Leeuwarden, The Netherlands

${ }^{c}$ Interventional Molecular Imaging, Department of Radiology,

Leiden University and Medical Centre, 2300 RC Leiden, The Netherlands

$\dagger$ Electronic supplementary information (ESI) available. See DOI: 10.1039/ c9cc03791h
}

PDMS stamps and lateral spreading of inks during printing. ${ }^{6,7}$ Moreover, a clean room is often required for reaching sub$10 \mu \mathrm{m}$ resolution feature size within UV-lithography and therefore also for $\mu \mathrm{CP}$. To circumvent the limitations of $\mu \mathrm{CP}$, alternative surface patterning techniques were developed such as dip-pen nanolithography, ${ }^{8,9}$ UV-initiated photografting, ${ }^{10,11}$ and laser ablation through light induced molecular adsorption technology. ${ }^{12,13}$ The wide adoption of these methodologies, however, may be limited by production costs.

As an alternative, plasma initiated patterning/plasma microcontact patterning $(\mathrm{P} \mu \mathrm{CP})$ was developed by Langowski et al. ${ }^{14}$ and Picone et al. ${ }^{15}$ for creating patterns of physisorbed proteins on surfaces. The high energetic plasma decomposes organic molecules on the surface, ${ }^{16-19}$ and a patterned PDMS stamp acts as a mask to control the exposure of the surface to the plasma. In contrast to plasma glass etching, which relies on $\mathrm{SF}_{6}, \mathrm{C}_{4} \mathrm{~F}_{8}$, or $\mathrm{CHF}_{3}$ as a fluorine based etchant for removing $\mathrm{Si}$ atoms, ${ }^{20}$ in this case, air is used as etching gas and the plasma decomposes only the organic molecules present on the surface, in a process known as isotropic 'chemical etching. ${ }^{21}$ So far, the $\mathrm{P} \mu \mathrm{CP}$ technique has been predominantly used for creating patterns of physisorbed molecules for biological applications. We hypothesized that this concept is more versatile and can also be used to obtain patterns on covalently coated surfaces. To demonstrate the potential of this approach, we focused on covalent surface modification starting from the organosilane monolayer assembly. The P $\mu \mathrm{CP}$ step can already be applied after organosilane modification or at a later stage to create patterns of molecules by protecting part of the surface during plasma treatment with a patterned PDMS stamp (Fig. 1). Further modification steps can also be carried out on the patterned surface and characterization is possible through labeling with specific targeting fluorophores for analysis of the surface by (fluorescence) microscopy. The ability to use $\mathrm{P} \mu \mathrm{CP}$ for patterning covalently attached monolayers could open the field of surface functionalization without the lengthy process of $\mu \mathrm{CP}$ optimization or using complicated and/or expensive techniques. 


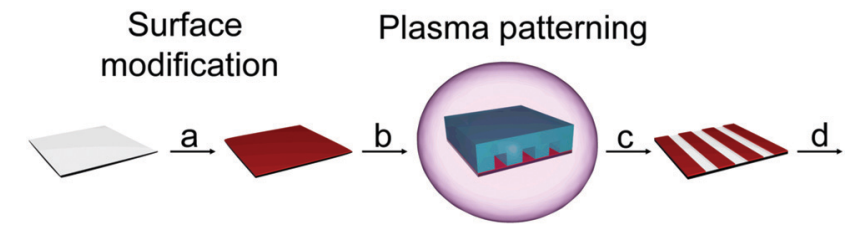

Fig. 1 Scheme showing principle of $\mathrm{P \mu CP}$ on surfaces: (a) (chemical) covalent glass slide modification with desired functionality, (b) placing of PDMS mask on modified slide for plasma patterning, (c) localized removal of surface functionality using plasma etching to yield patterns on glass surface, and (d) further surface functionalization steps.

We investigated the use of $\beta$-cyclodextrin $(\beta-\mathrm{CD})$ on glass surfaces as a model for the covalent attachment of monolayers and creating patterns using $\mathrm{P} \mu \mathrm{CP}$ methodology. The multiple steps for obtaining a monolayer of $\beta$-CD are well studied in literature, known as the 'molecular printboard',22,23 in which covalently, surface bound $\beta$-CD has been used for immobilizing non-covalently guest molecules ${ }^{2,4,24,25}$ and nanoparticles.,26 Within the field of supramolecular host-guest chemistry, adamantane is often used as a guest molecule because of its high affinity for $\beta$-CD due to its hydrophobic nature, size, and possibility for multivalent interactions. Therefore, the multiple steps for obtaining a monolayer of $\beta$-CD allows studying the effect of $\mathrm{P} \mu \mathrm{CP}$ on different molecular moieties, with respect to retaining the host-guest functionality of $\beta$-CD for the immobilization of adamantane functionalized molecules.

The $\beta$-CD glass surface functionalization works as displayed in Fig. 2a: (1) silanization of glass surface silanol groups using 3-aminopropyltriethoxysilane (APTES) for obtaining an amine layer, (2) covalent attachment of heptakis amino $\beta$-CD addition via 1,4-phenylene diisothiocyanate (PDITC) cross-linking, and (3) visualization of $\beta$-CD patterns with fluorescent divalent guest molecule cyanine 5-diadamantane $\left(\mathrm{Cy} 5-\mathrm{Ad}_{2}\right) \cdot{ }^{27} \mathrm{P} \mu \mathrm{CP}$ was carried out after the functionalization of the glass slide with APTES (Exp. 1), after the attachment of $\beta$-CD (Exp. 2), and also after the non-covalent immobilization of Cy5-Ad 2 (Exp. 3). $\$$ For Exp. 1-2, Cy5-Ad ${ }_{2}$ dye was subsequently added to functionalized surfaces to label the $\beta$-CD patterns. Each single experiment was then validated using fluorescence microscopy. In addition, we also created more complex patterns using $\mathrm{P} \mu \mathrm{CP}$ on fully functionalized $\beta$-CD surfaces which also generated sub-10 $\mu \mathrm{m}$ sized features. Importantly, all these experiments, including the fabrication of patterned silicon wafers for the PDMS stamps, were carried out without the use of a clean room.

For Exp. 1, after APTES monolayer assembly and creation of patterns through $\mathrm{P} \mu \mathrm{CP}$, the surface was first validated by addition of rhodamine $\mathrm{B}$ isothiocyanate (RITC), as an additional control (Fig. S1, ESI $\dagger$ ). RITC is highly reactive towards amines and can therefore be used to fluorescently label APTES patterns. Patterns $(150 \mu \mathrm{m})$ of bright fluorescent lines were observed, while dark regions $(50 \mu \mathrm{m})$ show no fluorescence as they were exposed to plasma treatment before addition of RITC. This indicated that plasma etching effectively removed all covalently bound APTES from the glass surface outside of the PDMS covered areas and that the protected APTES molecules a
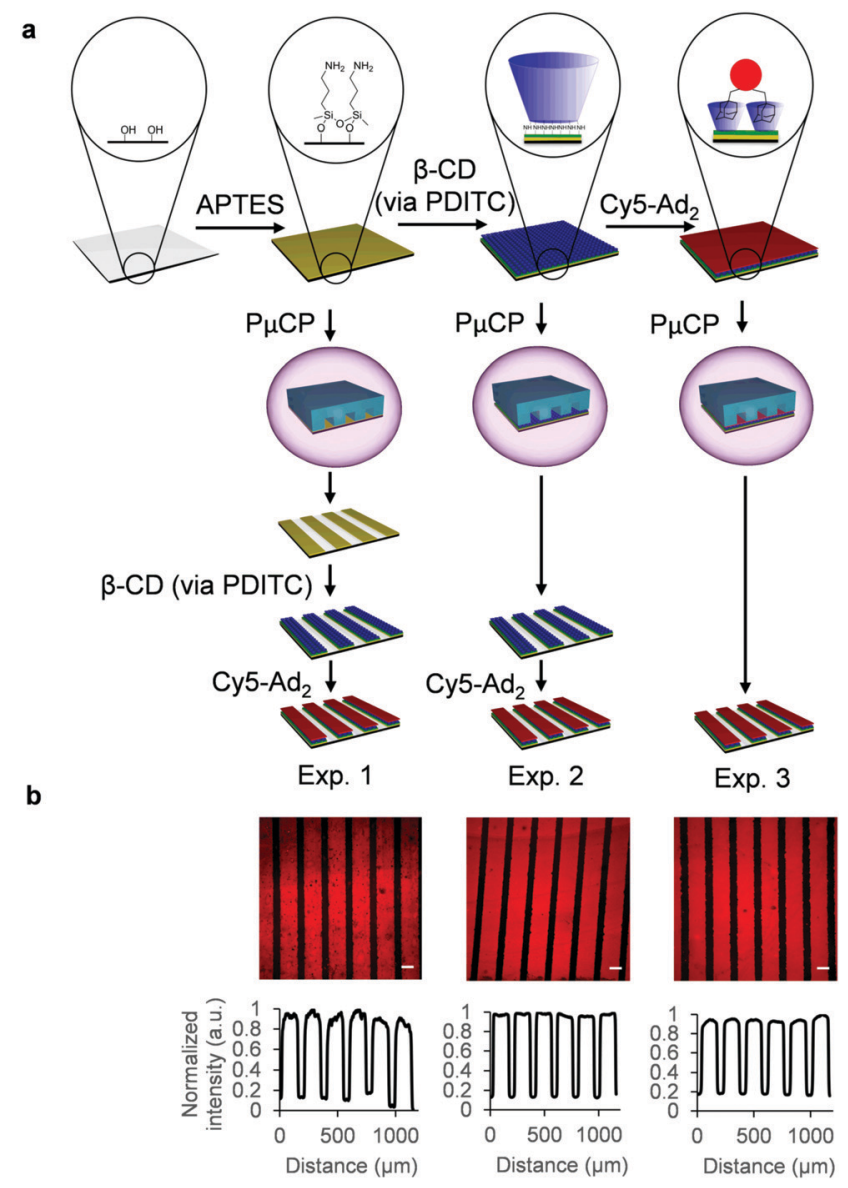

Fig. 2 (a) Scheme showing the $\beta$-CD glass surface functionalization steps and immobilization of fluorescent guest molecule Cy5-Ad $2 . P \mu C P$ was applied either after APTES monolayer assembly (Exp. 1), $\beta-C D$ attachment (Exp. 2), or after Cy5-Ad 2 immobilization (Exp. 3). (b) Fluorescence microscope images of Exp. 1: Cy5- $\mathrm{Ad}_{2}$ addition to $\beta$-CD after PDITC mediated attachment of $\beta-C D$ to P $\mu C P$ APTES patterns, Exp. 2: Cy5- $\mathrm{Ad}_{2}$ addition to $P \mu C P \beta-C D$ after PDITC mediated attachment of $\beta-C D$ to fully covered APTES surface, and Exp. 3: $\mathrm{P} \mu \mathrm{CP}$ of $\mathrm{Cy} 5-\mathrm{Ad}_{2}$ after its immobilization to fully covered $\beta$-CD surface. Profile plots showing the gray value intensity vs. the distance in $\mu \mathrm{m}$ are depicted below the fluorescence images and are corrected for beam curvature (see Fig. S2-S4 for explanation, ESI $\dagger$ ). Microscope images were captured with $10 \times$ objective and scale bars are $100 \mu \mathrm{m}$

were still functional. The patterned APTES surfaces were further reacted with PDITC and consecutively with heptakis amino $\beta$-CD (Fig. 2a). Addition of the fluorophore Cy5- $\mathrm{Ad}_{2}$ was carried out to visualize surface bound $\beta$-CD through host-guest interactions. Also in this case, clear fluorescent lines $(150 \mu \mathrm{m})$ of $\mathrm{Cy} 5-\mathrm{Ad}_{2}$ immobilized to $\beta$-CD patterns were observed (Exp. 1 , Fig. 2b, larger overview Fig. S6, ESI $\dagger$ ). Dark regions were again observed between $\beta$-CD lines, which indicates that $\beta$-CD was successfully cross-linked to APTES patterns.

In Exp. 2, fully covered surfaces of $\beta$-CD were P $\mu$ CP treated and subsequently incubated with $\mathrm{Cy} 5-\mathrm{Ad}_{2}$ (Fig. 2a). The selective immobilization of $\mathrm{Cy} 5-\mathrm{Ad}_{2}$ is clearly observed on $\beta$-CD patterns as bright fluorescent lines (Exp. 2, Fig. 2b, larger overview in Fig. S6, ESI $\dagger$ ). In Exp. 3, we used P $\mu$ CP in the final step of the surface modification process after addition of 
Cy5-Ad 2 (Fig. 2a). Clear patterns of host-guest immobilized Cy5- $\mathrm{Ad}_{2}$ are also observed in Fig. 2b for Exp. 3. The generated patterns for experiments 1-3 all show clear contrast between the desired feature and the spacing, which showcases the reproducibility of the technique. Features of fabricated PDMS stamps were compared with the resulting $\mathrm{P} \mu \mathrm{CP}$ patterns through repeating Exp. 3 from Fig. 2a and leaving the PDMS stamp on the surface during microscope imaging (Fig. S7, ESI $\dagger$ ). These images show that the fluorescent patterns are in accordance with the PDMS stamp features when comparing the Cy5 channel with the brightfield channel, which is also further confirmed with an overlay image (Fig. S8, ESI $\dagger$ ). Therefore, using $\mathrm{P} \mu \mathrm{CP}$ methodology for creating patterns of covalently attached monolayers is successful at multiple steps within a functionalization protocol allowing great versatility.

We also explored a simple approach, using $\mathrm{P} \mu \mathrm{CP}$, for creating intricate and smaller $\beta$-CD patterns than the $150 \mu \mathrm{m}$ line features of the applied PDMS stamp. In general, the fabrication of sub-10 $\mu \mathrm{m}$ patterns on surfaces through conventional techniques, such as UV-lithography, usually requires a clean room. Here, 'cross'-P $\mu \mathrm{CP}$ can be used to create unconventional small features, starting from conventional large patterns. After obtaining a fully functionalized $\beta$-CD surface according to the protocol shown in Fig. 2a, Exp. 2, the P $\mu \mathrm{CP}$ step for creating patterns was slightly altered by rotating the PDMS mask between multiple plasma cycles (Fig. 3a). The first plasma treatment step was carried out, as previously, by placing the PDMS stamp on the functionalized surface at $0^{\circ}$ (vertical lines). This step was then followed by several rotations of the PDMS stamp between plasma cycles: $\pm 90^{\circ}, \pm 45^{\circ}$, and finally $\pm 90^{\circ}$, all with respect to the previous step. This was done in order to remove more $\beta$-CD molecules from the surface through plasma etching. Cy5- $\mathrm{Ad}_{2}$ addition to the treated surface then yielded a variety of shapes when imaged on the microscope (Fig. 3b). As observed in Fig. 3b(iv), we also managed to fabricate distinguishable sub-10 $\mu \mathrm{m}$ features of $\beta$-CD on the same surface by using cross-P $\mu \mathrm{CP}$. The result shows clear contrast between functionalized and non-functionalized areas and are agreeable with the expected patterns depicted in the scheme in Fig. 3a.

In this work, we described how a micropatterning technique $(\mathrm{P} \mu \mathrm{CP})$ based on the spatially controlled removal of molecules through plasma treatment and PDMS stamps, can easily be applied to monolayers of covalently bound molecules on surfaces. We show that $\mathrm{P} \mu \mathrm{CP}$ can be used to create patterns during different steps and thus on different molecular moieties within the extensive $\beta$-CD surface functionalization, while also retaining the reactivity of the amine monolayer and the supramolecular functionality of the $\beta$-CD layer. We believe that, owing to its simplicity, $\mathrm{P} \mu \mathrm{CP}$ is more straightforward and reproducible than current micropatterning techniques for covalently bound

a

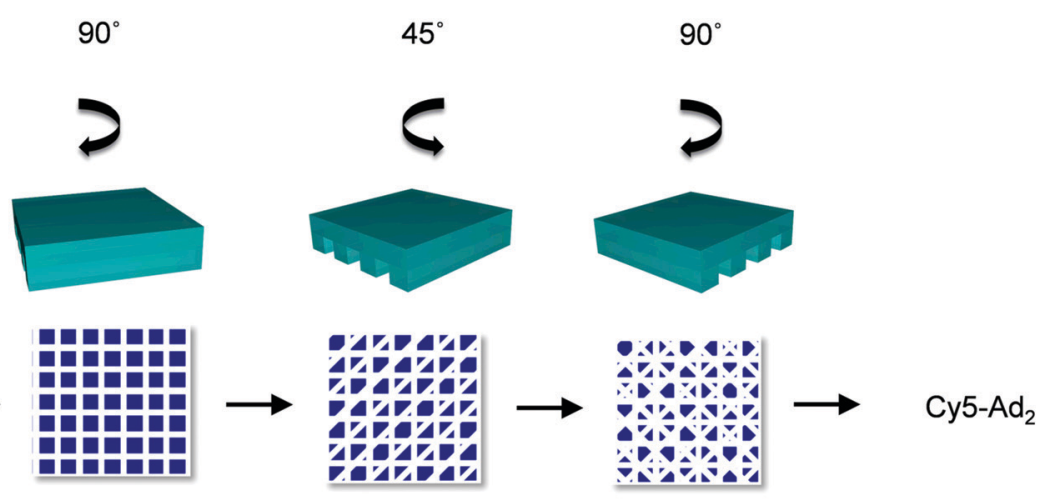

b
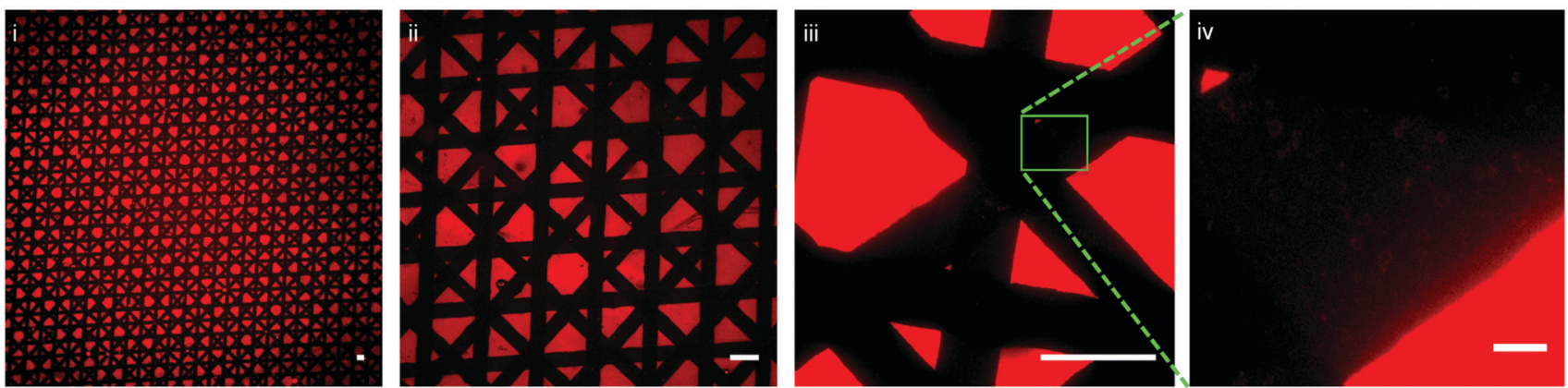

Fig. 3 (a) Scheme showing the concept of changing the stamp orientation in between P $\mu$ CP cycles on modified glass surface. The expected outcome of patterns on top of the glass surface is shown below the PDMS stamps. Cy5- $\mathrm{Ad}_{2}$ is added only after all 'cross'-P $\mu \mathrm{CP}$ steps. (b) Fluorescence microscope images of $\beta-C D$ surface after different $P \mu C P$ treatments and addition of $\mathrm{Cy} 5-\mathrm{Ad}_{2}$. Images were captured on the same glass slide with (i) $2.5 \times$ objective, (ii) $10 \times$ objective and (iii) $40 \times$ objective. A digital zoom of (iii) is depicted in (iv) to showcase a triangular shape with a size around $5 \mu$ m. All scale bars except for (iv) are $100 \mu \mathrm{m}$. The scale bar in (iv) is $10 \mu \mathrm{m}$. 
molecules. Moreover, by using 'cross'-P $\mu \mathrm{CP}$, a methodology which works contrapositive to additive manufacturing techniques, we fabricated sub-10 $\mu \mathrm{m}$ features. This simple technique can also open the concept of micropatterning to chemists who do not have access or experience in clean-room technology or microfabrication.

This work was performed in the cooperation framework of Wetsus, European Centre of Excellence for Sustainable Water Technology (www.wetsus.eu). Wetsus is co-funded by the Dutch Ministry of Economic Affairs and Climate Policy, the Province of Fryslân, and the Northern Netherlands Provinces. The authors like to thank the members of the research theme Source Separated Sanitation for the shared knowledge and financial support. We acknowledge Mark Rood for preparing the $\mathrm{Cy} 5-\mathrm{Ad}_{2}$ dye and Jan Bart ten Hove for input on experimental work.

\section{Conflicts of interest}

There are no conflicts to declare.

\section{Notes and references}

\$ Patterning isothiocyanate moieties through $\mathrm{P} \mu \mathrm{CP}$ was unsuccessful. We hypothesize that these groups are too reactive to remain intact for a prolonged duration and should be reacted soon after this modification step.

1 Y. Ahn, Y. Jang, N. Selvapalam, G. Yun and K. Kim, Angew. Chem., Int. Ed., 2013, 52, 3140-3144.

2 A. Mulder, S. Onclin, M. Peter, J. P. Hoogenboom, H. Beijleveld, J. ter Maat, M. F. Garcia-Parajo, B. J. Ravoo, J. Huskens, N. F. van Hulst and D. N. Reinhoudt, Small, 2005, 1, 242-253.

3 D. Dorokhin, S.-H. Hsu, N. Tomczak, D. N. Reinhoudt, J. Huskens, A. H. Velders and G. J. Vancso, ACS Nano, 2010, 4, 137-142.

4 A. Gonzalez-Campo, S. H. Hsu, L. Puig, J. Huskens, D. N. Reinhoudt and A. H. Velders, J. Am. Chem. Soc., 2010, 132, 11434-11436.

5 Y. Xia and G. M. Whitesides, Angew. Chem., Int. Ed., 1998, 37, 550-575.
6 J. N. Lee, C. Park and G. M. Whitesides, Anal. Chem., 2003, 75, 6544-6554.

7 A. Perl, D. N. Reinhoudt and J. Huskens, Adv. Mater., 2009, 21, 2257-2268.

8 R. D. Piner, J. Zhu, F. Xu, S. Hong and C. A. Mirkin, Science, 1999, 283, 661-663.

9 C.-C. Wu, D. N. Reinhoudt, C. Otto, V. Subramaniam and A. H. Velders, Small, 2011, 7, 989-1002.

10 D. Zahner, J. Abagat, F. Svec, J. M. J. Fréchet and P. A. Levkin, Adv. Mater., 2011, 23, 3030-3034.

11 F. L. Geyer, E. Ueda, U. Liebel, N. Grau and P. A. Levkin, Angew. Chem., Int. Ed., 2011, 50, 8424-8427.

12 P.-O. Strale, A. Azioune, G. Bugnicourt, Y. Lecomte, M. Chahid and V. Studer, Adv. Mater., 2015, 28, 2024-2029.

13 J. Decock, M. Schlenk and J.-B. Salmon, Lab Chip, 2018, 18, 1075-1083.

14 B. A. Langowski and K. E. Uhrich, Langmuir, 2005, 21, 10509-10514.

15 R. Picone, B. Baum and R. McKendry, in Methods in Cell Biology, ed. M. Piel and M. Théry, Academic Press, 2014, vol. 119, pp. 73-90.

16 K. Terpilowski and D. Rymuszka, Glass Phys. Chem., 2016, 42, 535-541.

17 T. Homola, J. Matoušek, M. Kormunda, L. Y. L. Wu and M. Černák, Plasma Chem. Plasma Process., 2013, 33, 881-894.

18 R. L. DeRosa, P. A. Schader and J. E. Shelby, J. Non-Cryst. Solids, 2003, 331, 32-40.

19 A. Alam, M. Howlader and M. Deen, J. Micromech. Microeng., 2014, 24, 035010.

20 K. Kolari, V. Saarela and S. Franssila, J. Micromech. Microeng., 2008, 18, 064010.

21 G. Nageswaran, L. Jothi and S. Jagannathan, in Non-Thermal Plasma Technology for Polymeric Materials, ed. S. Thomas, M. Mozetič, U. Cvelbar, P. Špatenka and K. M. Praveen, Elsevier, 2019, pp. 95-127.

22 M. J. W. Ludden, D. N. Reinhoudt and J. Huskens, Chem. Soc. Rev., $2006,35,1122-1134$.

23 S. Onclin, A. Mulder, J. Huskens, B. J. Ravoo and D. N. Reinhoudt, Langmuir, 2004, 20, 5460-5466.

24 C. A. Nijhuis, F. Yu, W. Knoll, J. Huskens and D. N. Reinhoudt, Langmuir, 2005, 21, 7866-7876.

25 J. Huskens, M. A. Deij and D. N. Reinhoudt, Angew. Chem., Int. Ed., 2002, 41, 4467-4471.

26 V. Mahalingam, S. Onclin, M. Peter, B. J. Ravoo, J. Huskens and D. N. Reinhoudt, Langmuir, 2004, 20, 11756-11762.

27 M. T. M. Rood, S. J. Spa, M. M. Welling, J. B. ten Hove, D. M. van Willigen, T. Buckle, A. H. Velders and F. W. B. van Leeuwen, Sci. Rep., 2017, 7, 39908. 